\title{
Autoimmune hepatitis, Wilson's disease, or both? An analysis of challenging cases
}

\author{
Magdalena Naorniakowska', Małgorzata Woźniak², Maciej Pronicki², Wiesława A. Grajkowska², \\ Diana Kamińska', Wojciech Jańczyk' ${ }^{1}$ Maciej Dądalski' ${ }^{1}$ Bożena Cukrowska², Piotr Socha' \\ 'Department of Gastroenterology, Hepatology, Nutritional Disorders and Paediatrics, The Children's Memorial Health \\ Institute, Warsaw, Poland \\ ${ }^{2}$ Department of Pathology, The Children's Memorial Health Institute, Warsaw, Poland
}

\section{ABSTRACT}

Introduction: The comorbidity of autoimmune hepatitis (AIH) and Wilson's disease (WD) can be considered, but has never been proven based on therapeutic response. There is a risk of misdiagnosis with standard diagnostic approaches. We describe a case of a challenging patients where final diagnosis could not be established with primary diagnostic approaches, and we question the coexistence of AIH and WD.

Material and methods: We identified four cases among 165 patients with WD and 321 with AIH treated in our hospital, whose primary diagnosis was changed or questioned, analysis of which shows the difficulty in differentiating between these two diseases.

Results: We presented four patients - three of them were diagnosed because of elevated serum aminotransferases activity and one due to acute hepatic failure. The three children were initially diagnosed as AIH, but after meticulous screening, WD was diagnosed. In the fourth case, WD was diagnosed from the beginning, but some autoimmune features and an incomplete response to the treatment prompted us to use steroids to achieve full recovery. Conclusions: WD can usually be distinguished from $\mathrm{AIH}$, but in selected cases differential diagnosis is challenging. It seems to be extremely important to carry out a complete diagnostic set for WD in patients with initial diagnosis of AIH with poor response to steroids. In a large cohort of patients with WD and with AIH, only one seemed to have comorbidity, but even in this case AIH was not fully confirmed.

KEY WORDS:

hepatitis, comorbidity, differential diagnosis, autoantibodies.

\section{INTRODUCTION}

Wilson's disease (WD) is an autosomal-recessive human copper-storage disorder caused by mutations within the $A T P 7 B$ gene located in chromosome 13 with an incidence in most populations of one in 30,000 [1]. It can present with variable symptoms (hepatic and neuropsychiatric). Establishing the diagnosis of WD is straightforward if major clinical and laboratory features are present, but can be difficult in some cases $[2,3]$.
Autoimmune hepatitis (AIH) is a progressive inflammatory liver disorder characterised serologically by high levels of aminotransferases (TA) and immunoglobulin G (IgG), presence of autoantibodies, and histologically by interface hepatitis when other aetiologies (e.g. viral, metabolic) were excluded $[4,5]$.

Both AIH and WD can present as acute fulminant or chronic hepatitis. The diagnosis of AIH is based on disease criteria and exclusion of WD. Comorbidities can be also considered but, even if such claims exist in the liter-

\section{ADDRESS FOR CORRESPONDENCE:}

Magdalena Naorniakowska, Department of Gastroenterology, Hepatology, Nutritional Disorders and Paediatrics, The Children's Memorial Health Institute, 20 Dzieci Polskich Ave., 04-730 Warsaw, Poland, ORCID: 0000-0002-5502-4208, e-mail: m.naorniakowska@ipczd.pl 
ature, they have never been proven based on therapeutic response. Sometimes, there is a significant risk of misdiagnosis, even if the standard diagnostic approaches were applied. Differential diagnosis or comorbidities of WD and $\mathrm{AIH}$ are claimed to be challenging and extremely important - still, the difficulties have never been described thoroughly based on clinical reports of several cases.

We describe challenging patients in whom the ultimate diagnosis of one of the diseases could not be established with the primary diagnostic approach, and we question the coexistence of AIH and WD.

\section{MATERIAL AND METHODS}

From 1996 to January 2017, in our Department of Gastroenterology, Hepatology, Nutritional Disorders and Paediatrics, we diagnosed WD in 161 children, and we identified 321 patients with AIH. The diagnosis of WD was based on the Ferenci scoring system $[6,7]$ with confirmation by mutation analysis in most cases. AIH was identified based on the elevated activity of aminotransferases, the level of IgG, the presence of auto-antibodies and histological changes (interface hepatitis) with exclusion of other aetiologies.

Among 161 patients with WD and 321 patients with $\mathrm{AIH}$, we identified four cases with WD, treated in our hospital, in whom primary diagnosis was changed or questioned which shows the difficulty in differentiating between these two diseases.

Data were collected from patients' medical histories and analysed retrospectively. The Local Ethics Committee approved the study.

\section{RESULTS}

\section{PATIENT 1}

A 13-year-old girl was admitted to a local hospital due to stomach pain. Laboratory tests were performed and revealed high activity of TA and gamma-glutamyl-

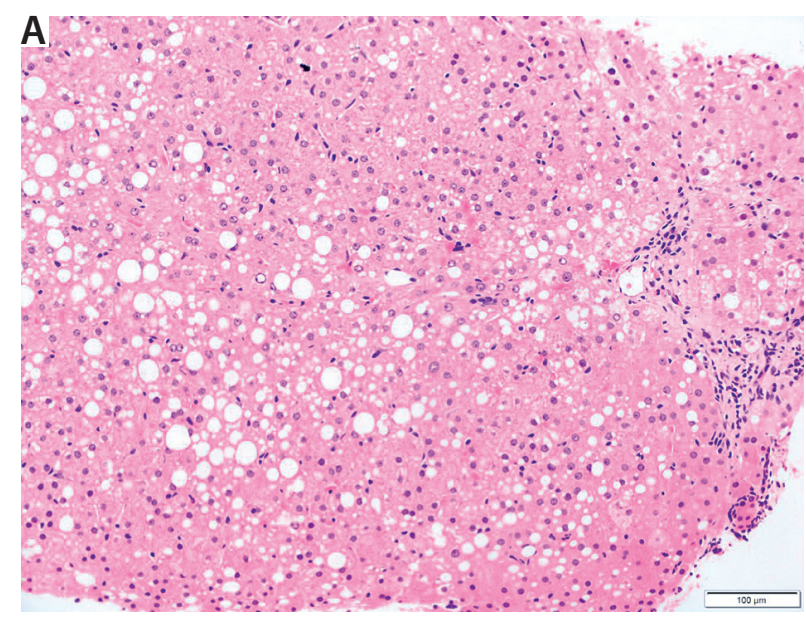

FIGURE 1. Patient 1 transferase (GGTP) and high levels of proteins, gamma globulins $(14.98 \mathrm{~g} / \mathrm{l})$. At that time the child was monitored without therapy, and after 7 months she was admitted again for further examination. AT and GGTP activity were still elevated: aspartate aminotransferase (AspAT) $83 \mathrm{U} / \mathrm{l}$, alanine aminotransferase (AlAT) 123 U/1, GGTP 69 U/1. Hepatomegaly was diagnosed by ultrasound (US). Viral hepatitis was excluded. Liver biopsy was performed and - based on liver histology (steatohepatitis and fibrosis - Fig. 1), positive antinuclear antibodies (ANA) and an elevation of IgG (Table 1) - AIH was diagnosed. Prednisone therapy was initiated, but it was not effective, and after 1 month azathioprine (AZT) was added to the therapy. After six months of unsuccessful treatment a second biopsy was done. It revealed the progression of steatosis and chronic inflammation (Table 2). Nevertheless, the therapy was continued. After one year from the onset of the first symptoms, the girl was admitted to our clinic and was tested for WD which was diagnosed based on her ceruloplasmin level, 24-hour urinary copper excretion, liver-copper concentration and a molecular genetic test (Ferenci score - FS: 10 points). Penicillamine therapy was introduced, and glucocorticosteroids (GC's) and AZT were withdrawn, leading to normal liver function tests.

\section{PATIENT 2}

An 11-year-old boy was admitted to a local hospital because of stomach pain and vomiting. In laboratory tests unconjugated hyperbilirubinemia was observed and hepatomegaly with portal hypertension was diagnosed by US. In gastroscopy erosive gastritis due to Helicobacter pylori infection was diagnosed and proper treatment was initiated. In repeated laboratory tests TA activity was elevated (AspAT 313 U/l, AlAT 231 U/l). Viral causes were excluded, and the patient was transferred to our clinic for further investigation. He primarily did not fulfil the Ferenci criteria for WD (Table 1), however liver copper content determination was not carried out at that time.

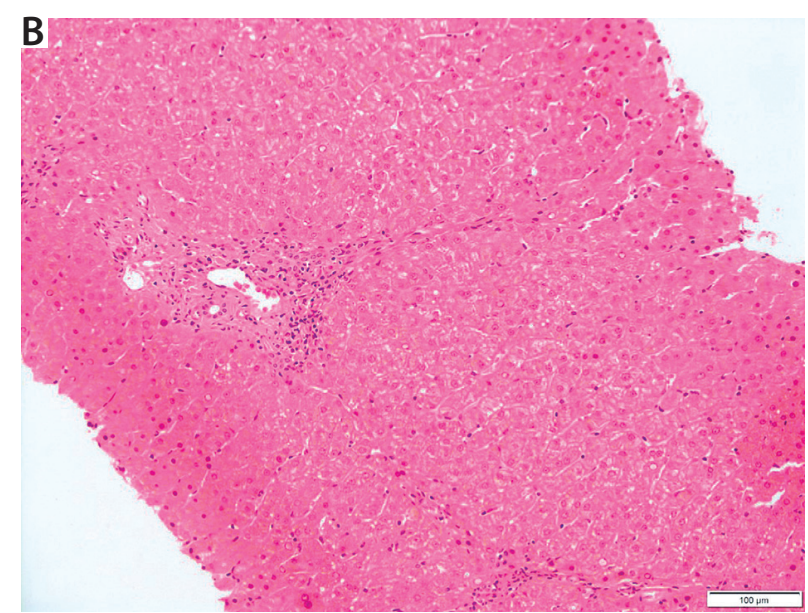


TABLE 1. Diagnostic tests and response to therapy

\begin{tabular}{|c|c|c|c|c|}
\hline \multirow[t]{2}{*}{ Parameter } & \multicolumn{4}{|c|}{ Patients } \\
\hline & 1 & 2 & 3 & 4 \\
\hline Diagnosis of AlH & $\begin{array}{c}\text { ANA } 1: 640 \\
\text { Gamma-globulins } \\
16.2 \mathrm{~g} / \mathrm{l} \\
\text { IgG } 2824.5 \mathrm{mg} \% \\
\text { Inflammation, cirrhosis }\end{array}$ & $\begin{array}{c}\text { ANA } 1: 640, \\
\text { ASMA } 1: 160 \\
\text { IgG }- \text { WNL } \\
\text { Severe inflammation, } \\
\text { severe fibrosis }\end{array}$ & $\begin{array}{c}\text { ANA } 1: 320 \\
\text { ASMA 1:80 } \\
\text { IgG }- \text { WNL } \\
\text { Periportal inflammation, } \\
\text { severe steatosis }\end{array}$ & $\begin{array}{l}\text { ANA } 1: 40 \\
\text { ASMA } 1: 40 \\
\text { IgG } 15.4 \mathrm{~g} / \mathrm{l} \\
\text { Steatohepatitis } \\
\text { and fibrosis }\end{array}$ \\
\hline $\begin{array}{l}\text { Primary exclusion } \\
\text { of WD }\end{array}$ & Tests not performed & $\begin{array}{c}\text { CP } 16 \mathrm{mg} / \mathrm{dl} \\
\text { Urine copper } 23 \mu \mathrm{g} / 24 \mathrm{~h}\end{array}$ & $\begin{array}{c}\mathrm{CP} 24 \mathrm{mg} / \mathrm{dl} \\
\text { Urine copper } 50 \mu \mathrm{g} / 24 \mathrm{~h}\end{array}$ & - \\
\hline $\begin{array}{l}\text { Final diagnosis } \\
\text { of WD }\end{array}$ & $\begin{array}{l}\quad \mathrm{CP}<7 \mathrm{mg} / \mathrm{dl} \\
\text { Urine copper } 151.2 \mu \mathrm{g} \\
\text { Liver tissue } 736.8 \mu \mathrm{g} / \mathrm{g} \\
2 \text { mutations }\end{array}$ & $\begin{array}{c}\text { Neurological symptoms } \\
\text { CP } 19 \mathrm{mg} / \mathrm{dl} \\
1 \text { mutation }\end{array}$ & $\begin{array}{l}\text { CP } 14 \mathrm{mg} / \mathrm{dl} \\
\text { Liver tissue } 879 \mu \mathrm{g} / \mathrm{g} \\
2 \text { mutations }\end{array}$ & $\begin{array}{c}\text { CP } 16 \mathrm{mg} / \mathrm{dl} \\
\text { Urine copper } 4901 \mu \mathrm{g} \\
2 \text { mutations } \\
\text { KF ring }\end{array}$ \\
\hline $\begin{array}{l}\text { Response } \\
\text { to steroids/AZT }\end{array}$ & GC's - slight & Yes & GC's - slight & Yes \\
\hline $\begin{array}{l}\text { Response to Zn, } \\
\text { D-p. or trientine }\end{array}$ & D-p. - yes & $\mathrm{Zn}$ - yes & D-p. - yes & $\begin{array}{l}\text { D-p. - yes } \\
\text { Than because } \\
\text { of neutropenia } \\
\text { - trientine }\end{array}$ \\
\hline $\begin{array}{l}\text { Steroid } \\
\text { withdrawing/tapered }\end{array}$ & Withdrawn & $\begin{array}{l}\text { Tapered (still getting } \\
\text { because of }(D)\end{array}$ & Withdrawn & No \\
\hline Comorbidities & - & Crohn disease & - & - \\
\hline
\end{tabular}

AlH - autoimmune hepatitis, ANA - antinuclear antibodies, ASMA - anti-smooth muscle antibody, lgG - immunoglobulin G, WNL - within normal limits, WD - Wilson's disease, CP - ceruloplasmin, AZT - azathioprine, GC's - glucocorticosteroids, KF-Kayser-Fleischer, Zn - zinc, D-p. - D-penicillamine, CD - Crohn's disease

TABLE 2. Liver histology described according to the modified Kleiner scoring system

\begin{tabular}{|l|c|c|c|c|c|c|c|c|c|}
\hline \multirow{2}{*}{ Parameter } & \multicolumn{9}{c|}{ Patient } \\
\cline { 2 - 11 } & \multicolumn{2}{|c|}{1} & \multicolumn{2}{c|}{2} & \multicolumn{2}{c|}{3} & \multicolumn{2}{c|}{4} \\
\hline Portal inflammation 0-3 & 2 & 1 & 2 & 0 & 1 & 1 & 0 & 2 & 0 \\
\hline Lobular inflammation 0-3 & 0 & 0 & 0 & 0 & 0 & 0 & 0 & 1 & 0 \\
\hline Macrovesicular steatosis 0-3 & 2 & 0 & 0 & 0 & 1 & 2 & 2 & 0 & 0 \\
\hline Microvesicular steatosis 0-3 & 2 & 0 & 0 & 0 & 1 & 3 & 2 & 1 & 0 \\
\hline Fibrosis 0-4 & 4 & 2 & 3 & 3 & 2 & 3 & 3 & 4 & 1 \\
\hline Cholestasis 0-3 & 0 & 0 & 0 & 0 & 0 & 0 & 0 & 0 & 0 \\
\hline
\end{tabular}

Modified Kleiner scoring system - semiquantitative scoring system used for non-alcoholic fatty liver disease

Based on the detection of typical auto-antibodies (ANA, anti-smooth muscle antibody - ASMA) and liver histology (severe inflammation and fibrosis - Fig. 2) (Table 2) prednisolone therapy was initiated. Gamma globulins and IgG levels were within normal limits. After one year the child developed psychiatric (depressive) and neurological symptoms (bradykinesia, gait abnormalities, visual acuity disturbances). Brain magnetic resonance imaging (MRI) revealed specific abnormalities (hypertensive focus in $\mathrm{T} 2$ images in the globus pallidus). Based on the ceruloplasmin level, neurological symptoms, and a molecular investigation, WD was diagnosed (FS: 4 points) and zinc therapy was initiated. Meanwhile, the patient was diagnosed with Crohn's disease. The patient was treated with zinc, AZT and 5-aminosalicylic acid (5-ASA) with a good clinical and laboratory response.

\section{PATIENT 3}

A 5-year-old girl was admitted to our clinic due to elevated activity of TA (AspAT 125 U/1, AlAT 205 U/1). Initially, she had been diagnosed in a local hospital, where viral causes, a1-antitrypsin deficiency and cystic fibrosis as well as WD were excluded (her serum ceruloplasmin level and 24-hour urine-copper excretion were within normal limits). In our hospital, repeated laboratory tests for WD revealed no abnormalities (Table 1). Liver biopsy was inconclusive (periportal inflammation, severe steatosis with no cholestasis - Fig. 3) (Table 2). However, due to elevated TA, positive autoantibodies (ANA $1: 320$, ASMA $1: 80$ ) and inflammation shown in liver biopsy, because other aetiology could not be established, prednisolone therapy was initiated. After one year of therapy, 

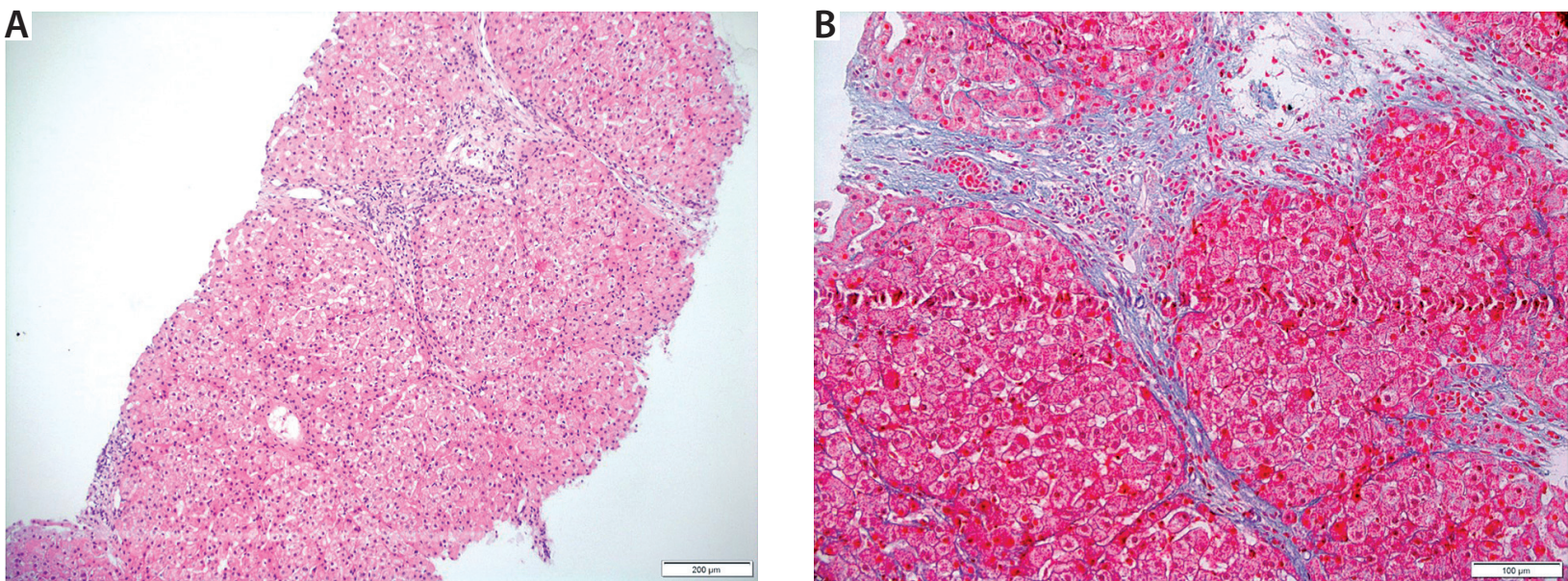

FIGURE 2. Patient 2
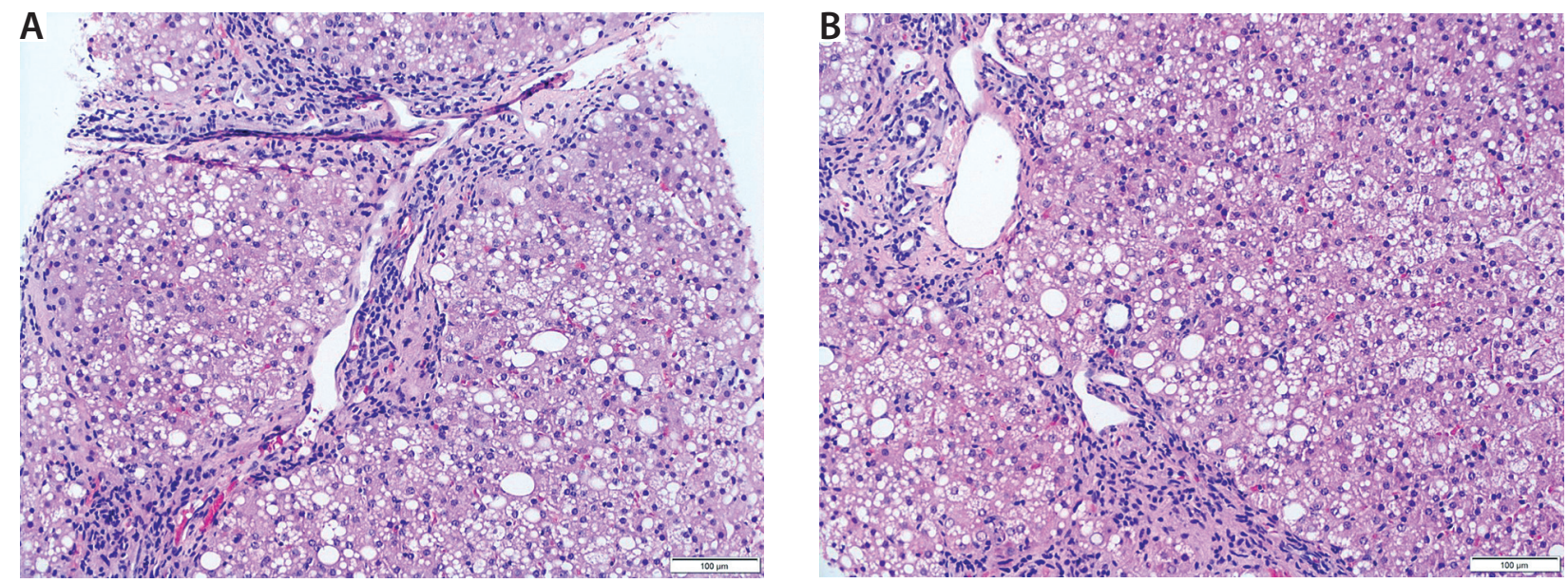

FIGURE 3. Patient 3

TA was still elevated. In the second diagnostic approach WD was diagnosed based on a low serum-ceruloplasmin level and increased liver-copper concentration, finally confirmed by a molecular test (FS: 7 points). Penicillamine therapy was initiated, and after one year steroids were withdrawn leading to normal liver function test results.

\section{PATIENT 4}

The last patient, a 15-year-old girl, was admitted to our clinic with acute hepatitis, and was primarily diagnosed with WD based on the ceruloplasmin level, 24-hour urinary copper excretion, presence of KayserFleischer rings, and a molecular test (FS: 9 points). Penicillamine therapy was started but one month later, because of thrombocytopenia and increased TA activity based on positive ANA $(1: 40)$ and ASMA $(1: 40)$ and elevated IgG $(15.4 \mathrm{~g} / \mathrm{l})$, steroids were added to the therapy (a liver biopsy could not be performed at that time because of clotting disturbances) with positive response. After the next 4 weeks, the administration of trientine was initiated, and penicillamine was withdrawn, due to

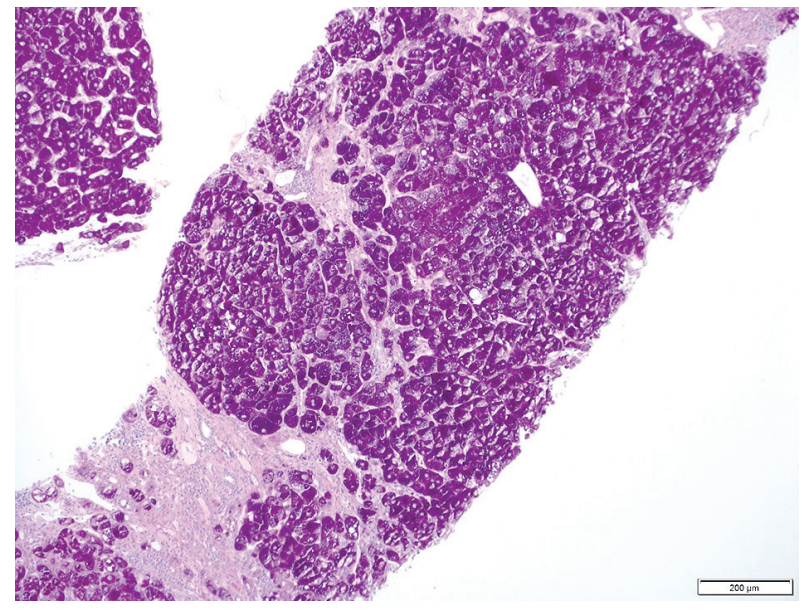

FIGURE 4. Patient 4

severe neutropenia. Liver biopsy was performed after the correction of coagulation disturbances and showed steatohepatitis and fibrosis (Fig. 4, Table 2). Six months later, we gradually reduced trientine and started zinc therapy. After the next 7 months, TA was slightly elevated again. Due to abnormalities indicated by laboratory tests, 
low-dose GCs therapy was resumed, and the patient was transferred to the hepatic outpatient clinic for adults (she was 18 years old).

\section{DISCUSSION}

In acute liver failure and in chronic hepatitis, especially in children, correct diagnosis and selecting the appropriate therapy still remains a challenge in selected patients in spite of advanced biochemical, histological and molecular techniques.

There were several reasons for the delayed diagnosis of WD in our cohort: the diagnosis was missed because the standard diagnostic tests for WD were not applied in the local hospital (patient 1), the biochemical basic copper metabolic tests did not indicate WD (ceruloplasmin and urinary copper excretion, patients 2 and 3 ) or the diagnosis of WD was established but comorbidity with AIH still could not be excluded.

Based on the experience of our clinic and case reports from other centres, it seems to be extremely important to perform differential diagnosis between AIH and WD. WD can be suspected in any child aged older than 1 year with evidence of liver dysfunction [3]. According to the recent recommendations, diagnostic testing for WD in suspected patients should include liver function tests (serum TA, conjugated and total bilirubin; alkaline phosphatase and prothrombin time/INR), serum ceruloplasmin, and 24-hour urinary copper excretion [3]. In fact, three of our patients were tested for WD and initially WD was excluded in two of them based on biochemical testing (only one child was not diagnosed according to the recommendations). Thus, we showed that WD should be retested in patients with poor response to steroid therapy, or in cases where neurological or psychiatric symptoms occur. We can also speculate that application of combined liver copper determination and molecular diagnosis would have allowed an earlier diagnosis of those two patients and that the recommended basic biochemical testing (ceruloplasmin and 24-hour urinary copper excretion ) [3] is not sufficient to differentiate between AIH and WD. In patients with clinical and laboratory-confirmed WD, and features of $\mathrm{AIH}$ at the same time, starting therapy can be considered only with medications for $\mathrm{WD}$, and then prednisone therapy may be considered.

Our results confirm the diagnostic value of the scoring system proposed by Ferenci et al., which includes clinical, biochemical, histological and molecular findings $[6,7]$. This system has already been proven to be reliable in children [8]. As indicated in our study, each of the diagnostic tests has its limitations and, therefore, there is no single test for the diagnosis of WD that would be reliable.

On the other hand, a simple and accurate diagnostic scoring system for AIH in children has not been established for some time. Criteria for diagnosis of AIH were formalised in 1993 and revised in 1999. Simplified criteria were developed in 2008 for adults only [9]. Mileti et al. presented a retrospective study which supported the usefulness of the Simplified Diagnostic Criteria for Autoimmune Hepatitis (2008) in children with AIH (except in those AIH patients with acute liver failure) with $87 \%$ sensitivity and $89 \%$ specificity. However, the group of patients was relatively small (37 children with AIH diagnosis and 40 children diagnosed with other liver diseases) and there were no patients with WD in the control group [10]. The latest diagnostic criteria for the diagnosis of juvenile AIH were published in 2018 by The European Society for Paediatric Gastroenterology Hepatology and Nutrition (ESPGHAN) Hepatology Committee - however it was not validated in children in comparison with other groups of patients [5]. We retrospectively put our patients on this scale [5] and none of our patients fulfilled the criteria of definite AIH (at the time of diagnosis the criteria were not available yet) the scores were as follows: patient $1-7$ points, patient 2 -5 points, patient $3-5$ points, patient $4-3$ points. According to Ferri et al., more studies with larger groups seem to be necessary to validate diagnostic criteria in paediatric patients [11]. In addition, unlike WD, AIH diagnosis cannot be verified by a simple single test like molecular analysis in WD.

There are only a few cases of simultaneous presentation of WD and AIH reported in the literature. Dara et al. described a boy with coexistence of these two diseases as declared by authors. WD was diagnosed based on the Ferenci scoring system (7 points) and AIH was diagnosed based on the Simplified Diagnostic Criteria for Autoimmune Hepatitis. The boy was treated with GCs, AZT and penicillamine with good clinical and laboratory results. However, there is no evidence that all the medications were necessary [12]. One may speculate that penicillamine therapy could have been sufficient.

Milkiewicz et al. presented two females, who were 15 and 23 years old, with classical features of WD and some features of AIH. In the first case, prednisolone therapy was initiated before the results of screening for WD became available. After the patient scored 5 points on the Ferenci scale penicillamine treatment was introduced, accompanied by the tapering of the GC's dose, with good results. The second patient had no proper diagnostic approach, but on the basis of the normal ceruloplasmin level (40 mg/dl) WD was excluded. AIH was diagnosed and prednisolone therapy was initiated with an initial improvement. However, after less than 3 years, the patient needed a liver transplant, and diagnostic tests performed at that time revealed the diagnosis of WD with 6 points on the Ferenci scale [13]. We may again speculate that both patients had only WD which initially was not properly diagnosed and $\mathrm{AIH}$ was a false diagnosis. In those cases, like in the case of our first patient, steroids were introduced as initial treatment and caused transient clinical improvement. 
Normal ceruloplasmin levels, which can occur in $20 \%$ of paediatric and adult patients with WD, can lead to a missed diagnosis what was observed in our patient number 3 [14]. On the other hand, hypo-ceruloplasminemia could also be observed in other hepatic disorders, such as decompensated liver disease, or in other chronic hepatopathies as a congenital glycosylation disorder [14]. Ilan et al. reported a case of a 17-year-old female with $\mathrm{AIH}$ and undetectable ceruloplasmin concentration (she did not meet other WD criteria) which increased to normal during steroid treatment [15]. Cauza et al. found 17 cases of hypo-ceruloplasminemia among 2867 screened patients with various liver diseases (excluding WD) [16].

Elevated titers of autoantibodies are an indicative of AIH but they are not specific as ANA and ASMA can also be detected in healthy children as well as in patients with other liver diseases, including WD [17]. To our knowledge, there are no systematic studies on autoantibodies in WD in children, but Gregorio et al. tested eighty children with non-autoimmune liver disease, including 20 patients with WD, for autoantibodies (ANA, ASMA, LKM-1). Four of them had ANA titers of $\geq 1: 40$ (20\%) and one patient had ASMA titers of $\geq 1: 40$. It seems that positive autoantibodies in WD can be found even if the diagnosis of AIH is not confirmed [18]. Autoantibody in WD can be induced by hepatocyte necrosis, especially in the early stages of this disease [12].

Neither histological hallmarks are sufficient to distinguish one disease from another. As no histopathological feature of WD is specific - microvesicular and macrovesicular steatosis, inflammation, fibrosis and cirrhosis, all are seen in other conditions - histopathological findings in the liver are not diagnostic criteria in WD $[14,19]$. However, liver steatosis should increase alertness and lead to wider testing for WD. In AIH, typical histopathological features are interface hepatitis, emperipolesis (active penetration by one cell into and through a larger cell), and hepatic rosette formation (each of the three features has to be present to be considered as typical). However, some patients with WD can demonstrate active inflammatory changes, even significant interface hepatitis with prominent plasma cells; in these cases distinction from AIH can be difficult [20]. Ultimately, in difficult cases, a liver biopsy should be performed, whenever possible, to complement the diagnostic approach and mark the liver-copper content [19].

Establishing the diagnosis in the setting of acute liver failure (ALF) is the most challenging and still critical due to differences in treatment; in WD chelator therapy can be effective, but the improvement occurs after a longer period of treatment, whereas patients with ALF due to AIH can respond to GC's. Moreover, there are different criteria for liver transplantation in the course of ALF due to WD and AIH [21]. Santos et al. described a 17-year-old girl with ALF who presented with clinical and histological features consistent with both WD and AIH. Because of the lack of conclusive data in support of WD, GC's therapy was started. However, the patient failed to respond. She underwent successful liver transplantation (before the diagnosis of WD could be confirmed). The authors pointed out the important role of correct diagnosis which may save lives [21]. A similar case was presented by Loudianos et al. - a 15-year-old girl with ALF and confirmed WD (11 FS points) also revealed some autoimmune features. Despite including treatment for both WD and AIH, her condition got worse and she underwent successful liver transplantation [22]. Those two cases show that ALF due to WD can present with AIH features, so patients presenting with ALF and positive auto-antibodies require a rapid diagnostic approach for WD.

In general, WD disease can be distinguished from $\mathrm{AIH}$ but in some cases differential diagnosis is challenging. In a big cohort of WD and AIH patients only one patient seemed to have comorbidity and even in this case AIH was not fully confirmed. Unfortunately, it is not uncommon to miss the diagnosis of WD in patients primarily diagnosed with $\mathrm{AIH}$, and to treat them with steroids and/or azathioprine. Therefore, it seems to be extremely important to carry out a complete diagnostic set for WD in patients with initial diagnosis of AIH (including molecular analysis) which is even more important when therapy for AIH fails.

\section{DISCLOSURE}

The authors declare no conflict of interest.

\section{REFERENCES}

1. Ala A, Walker AP, Ashkan K, et al. Wilson's disease. Lancet 2007; 369: 397-408.

2. Gow PJ, Smallwood RA, Angus PW, et al. Diagnosis of Wilson's disease: An experience over three decades. Gut 2000; 46: 415-419.

3. Socha P, Janczyk W, Dhawan A, et al. Wilson's Disease in Children: A position paper by the European Society for Paediatric Gastroenterology, Hepatology and Nutrition Committee. J Pediatr Gastroenterol Nutr 2018; 66: 334-344.

4. Mieli-Vergani G, Vergani D. Autoimmune hepatitis in childhood. Clin Liver Dis 2014; 3: 6-8.

5. Mieli-Vergani G, Vergani ÃD, Baumann U, et al. Diagnosis and Management of Paediatric Autoimmune Liver Disease: ESPGHAN Hepatology Committee Position Statement. J Pediatr Gastroenterol Nutr 2018; 66: 345-360.

6. Ferenci P, Caca K, Loudianos G, et al. Diagnosis and phenotypic classification of Wilson disease. Liver Int 2003; 23: 139-142.

7. Seo JK. Diagnosis of Wilson Disease in Young Children: Molecular Genetic Testing and a Paradigm Shift from the Laboratory Diagnosis. Pediatr Gastroenterol Hepatol Nutr 2012; 15: 197-209.

8. Nicastro E, Ranucci G, Vajro P, et al. Re-evaluation of the Diagnostic Criteria for Wilson Disease in Children With Mild Liver Disease. Hepatology 2010; 52: 1948-1956.

9. Hennes EM, Zeniya M, Czaja AJ, et al. Simplified criteria for the diagnosis of autoimmune hepatitis. Hepatology 2008; 48: 169-176. 
10. Mileti E, Rosenthal P, Peters MG. Validation and Modification of Simplified Diagnostic Criteria for Autoimmune Hepatitis in Children. Clin Gastroenterol Hepatol 2012; 10: 417-421.

11. Ferri PM, Ferreira AR, Miranda DM, et al. Diagnostic criteria for autoimmune hepatitis in children: A challenge for pediatric hepatologists. world J Gastroenterol 2012; 18: 4470-4473.

12. Dara N, Imanzadeh F, Sayyari AA, et al. Simultaneous Presentation of Wilson's Disease and Autoimmune Hepatitis; A Case Report and Review of Literature. Hepat Mon 2015; 15: 10-13.

13. Milkiewicz P, Saksena S, Hubscher SG, Elias E. Wilson's disease with superimposed autoimmune features: Report of two cases and review. J Gastroenterol Hepatol 2000; 15: 570-574.

14. Della Corte C, Mosca A, Vania A, et al. Pediatric liver diseases: current challenges and future perspectives. Expert Rev Gastroenterol Hepatol 2016; 10: 255-265.

15. Ilan Y, Hillman M, Oren R, et al. Undetectable caeruloplasmin values in a patient with autoimmune chronic active hepatitis. Gut 1991; 32: 549-550.

16. Cauza E, Maier-Dobersberger T, Polli C, et al. Screening for Wilson's disease in patients with liver diseases by serum ceruloplasmin. J Hepatol 1997; 27: 358-362.

17. Narkewicz MR, Horslen S, Belle SH, et al. Prevalence and Significance of Autoantibodies in Children With Acute Liver Failure. J Pediatr Gastroenterol Nutr 2017; 64: 210-217.

18. Gregorio GV, Davies ET, Mieli-Vergani G, Vergani D. Significance of extractable nuclear antigens in childhood autoimmune liver disease. Clin Exp Immunol 1995; 102: 308-313.

19. Dezsőfi A, Baumann U, Dhawan A, et al. Liver biopsy in children: position paper of the ESPGHAN Hepatology Committee. J Pediatr Gastroenterol Nutr 2015; 60: 408-420.

20. Johncilla M, Mitchell KA. Pathology of the Liver in Copper Overload. Semin Liver Dis 2011; 31: 239-244.

21. Santos RG, Alissa F, Reyes J, et al. Fulminant hepatic failure: Wilson's disease or autoimmune hepatitis? Implications for transplantation. Pediatr Transplant 2005; 9: 112-116.

22. Loudianos G, Zappu A, Lepori MB, et al. Acute Liver Failure Because of Wilson Disease With Overlapping Autoimmune Hepatitis Features. J Pediatr Gastroenterol Nutr 2016; 63: e23-24. 